\title{
Association Between Prehospital Airway Management Methods and Neurologic Outcome in Out-Of-Hospital Cardiac Arrest (OHCA) with Respiratory Cause.
}

\section{Yeongho Choi}

Seoul National University Hospital, Laboratory of Emergency Medical Service, Seoul National University Hospital

Tae Han Kim ( $\sim$ adoong01@snu.ac.kr)

Seoul National University Hospital https://orcid.org/0000-0003-3855-081X

\section{Ki Jeong Hong}

Seoul National University Hospital, Laboratory of Emergency Medical Service, Seoul National University Hospital

\section{Kyoung Jun Song}

Seoul National University Seoul Metropolitan Government Boramae Medical Center, Laboratory of Emergency Medical Service, Seoul National University Hospital

\section{Sang Do Shin}

Seoul National University Hospital, Laboratory of Emergency Medical Service, Seoul National University Hospital

\section{Original research}

Keywords: Out-of-hospital cardiac arrest, Emergency medical service, Advanced airway placement, Respiratory cause

Posted Date: March 31st, 2021

DOI: https://doi.org/10.21203/rs.3.rs-357226/v1

License: (9) (i) This work is licensed under a Creative Commons Attribution 4.0 International License. Read Full License 


\section{Abstract}

Purpose: Cardiac arrests are resulted by various aetiology including respiratory cause. Advanced airway placement is an important prehospital intervention for oxygenation and ventilation in respiratory cardiac arrest. We evaluated the association between of advanced airway method and neurologic outcome in arrest with respiratory cause.

Method: Adult witnessed non-traumatic OHCAs treated by emergency medical service (EMS) providers in 2013-2017 were enrolled in a nationwide OHCA database. The association between airway management methods (endotracheal intubation (ETI), supraglottic airway (SGA) and bag valve mask (BVM)) and outcome were evaluated according to the presumed cause of cardiac arrest (cardiac, respiratory or others). The primary outcome was good neurological recovery at discharge. Multivariable logistic regression models with interaction analysis was conducted.

Result: Of 40,443 eligible OHCA patients, the cause of arrest of $90.0 \%, 7.5 \%$, and $2.4 \%$ of patients were categorized as cardiac, respiratory and others, respectively. There were no statistically significant differences in the effect of the advanced airway type on good neurologic recovery in the total population (aOR 0.96 (0.81-1.14) for ETl; 1.01 (95\% Cl 0.93-1.11) for BVM). However, ETI was associated with better neurologic recovery than SGA or BVM in OHCA in cardiac arrest with suspected respiratory cause (aOR 3.12 (95\% Cl 1.24-7.80) for ETI; 0.99 (95\% Cl 0.51-1.91) for BVM).

Conclusion: Prehospital ETI was associated with good neurologic outcome when the cause of arrest was respiratory. ETI may be considered initially when a respiratory cause is suspected on the scene.

\section{Introduction}

Various etiologies are responsible for the occurrence of out-of-hospital cardiac arrest (OHCA). $(1,2)$ Although majority of OHCA is caused by cardiac cause, respiratory problems resulting from hypoxia and hypoventilation in conditions such as ARDS, pneumonia, COPD and asphyxia also cause cardiac arrest.(3-5) For cardiac arrest caused by respiratory aetiology, resuming and providing oxygenation and ventilation during cardiac arrest is especially important for correcting the cause of arrest and minimizing neurological damage in the brain. $(6,7)$

Advanced airway placement is one of the key prehospital interventions performed by emergency medical service (EMS) providers during field resuscitation of OHCA.(8) The main purpose of advanced airway placement is to provide more effective oxygenation and ventilation than basic airway such as bag-valve-mask $(B V M)$. $(9,10)$ Various studies have compared the effects of prehospital advanced airway management methods on outcomes in OHCA, but they are mostly tested in cardiac arrest with presumed cardiac cause the results are still controversial. (11-15)

Difference in bystander response, prehospital epinephrine administration and clinical outcome between arrests caused by respiratory and cardiac cause have been reported in other studies.(16-18)

However, little is known about the association of prehospital advanced airway management and outcome according to the cause of cardiac arrest.

In this study, we investigated the association between prehospital advanced airway method and neurologic outcome according to the presumed cause of cardiac arrest under the hypothesis that the effect of each advanced airway management type would be different when cause of arrest is suspected to be respiratory.

\section{Methods}

\section{Study design and setting}


A retrospective observational study was conducted using the prospectively collected nationwide EMS based OHCA registry of Korea. In Korea, a single national fire agency exclusively operates the prehospital EMS system. In cases of OHCA, EMS providers perform basic life support (BLS), including the use of automatic defibrillators for rhythm analysis and defibrillation. EMS providers have no authority to declare death or stop CPR unless there is return of spontaneous circulation (ROSC). Prehospital EMS providers in Korea are mostly emergency medical technicians (EMTs) and nurses. The majority of EMS providers are EMTs, for whom there are two levels of national certification (Level-1 EMT and Level-2 EMT). Level-1 EMTs provide services similar to EMT-intermediate services in EMS systems in United States. Advanced airway placement can only be performed by a Level-1 EMT or a nurse under direct medical control from a medical director on the phone. Endotracheal intubation or supraglottic airway insertion, such as l-gel and laryngeal mask airway (LMA), are also available. The decision to perform advanced airway placement and selection between airway techniques is usually at the discretion of an on-duty EMS provider.

\section{Data source}

The Nationwide OHCA database, which includes all EMS-assessed OHCAs, was retrieved from the following four sources: the EMS run sheets for basic ambulance operation information, the EMS cardiac arrest registry, the dispatcher CPR registry for the Utstein factors, and the hospital medical record review registry for hospital care and outcomes. A detailed description of the data acquisition of each registry, as well as the training and quality of the medical record reviewers, have been described in previous studies. $(19,20)$

\section{Study population}

All adult witnessed OHCAs assessed and treated by EMS providers from January 2013 to December 2017 were initially enrolled. OHCAs witnessed by EMTs during transport (whose selecting airway management method could different from bystander witnessed OHCA patients), OHCAs treated only by EMS teams consisting of level 2 EMTs only (who could not perform advanced airway placement) and OHCAs with unknown survival and neurologic outcome were excluded. We also excluded OHCAs with traumatic cause.

\section{Exposure and outcome variables}

The primary exposure of interest was the type of airway management method. The leader of the EMS team at the scene recorded a detailed OHCA registry, including the method of airway management. The leader of the team recorded whether the advanced airway was attempted, if it was successful (and if successful, the time of advanced airway placement) and the type of advanced airway method attempted (endotracheal intubation or supraglottic airway (Combitube, LMA, King airway or I-gel)). Current guidelines for prehospital EMS management recommend that level-1 EMTs or nurses can perform advanced airway placement according to their preference under direct medical direction. The guidelines recommend the procedure should take less than 30 seconds and that attempts should be performed up to 2 times. The primary outcome of the study was good neurological recovery at hospital discharge, defined as a cerebral performance category (CPC) 1 or 2 . Medical record reviewers assessed the CPC score based on discharge summary abstracts or documented medical records. Secondary outcome was survival to hospital discharge.

\section{Cause of cardiac arrest}

Pathogenesis of cardiac arrest in the database is determined by trained medical record reviewers after reviewing hospital records of patients according to Ustein recommendation.(21) In our analysis, we recategorized the cause of arrest into three groups after excluding traumatic arrest. Cause of arrest was presumed to be cardiac unless there was evidence to suggest a non-cardiac cause. Cardiac arrests caused by obvious aggravation of respiratory disease, asphyxia (including foreign body airway obstruction), drowning(submersion) or hanging were categorized as respiratory group. Lastly, remaining arrests by other etiologies such as poisoning or electrocution were categorized as others. We excluded traumatic arrest other than asphyxia, drowning and hanging from our study. 


\section{Statistical Analysis}

Demographics, EMS management, hospital management and outcomes were compared by cause of arrest using the $\chi 2$ test for categorical variables and one-way analysis of variance (ANOVA) or the Kruskal-Wallis test for continuous variables.

Multivariable logistic regression was conducted to analyze the effect of airway management method on the outcome and is presented as the odds ratios (ORs) with $95 \%$ confidence intervals $(\mathrm{Cl})$ after adjusting for covariables. The variables included in the model were age, gender, location of arrest, bystander CPR, response time interval, hypertension and heart disease. We tested for multicollinearity between the covariables in the model, and there was no multicollinearity. To assess changes in the effect of airway management according to the cause of arrest, we added an interaction analysis between airway management methods and the cause. STATA 14.1 (StataCorp, College Station, TX, USA) was used for all analyses.

\section{Results}

In total, 145,003 OHCA patients in our registry were initially enrolled from January 2013 to December 2017. Patients aged less than 18 years $(N=3,170)$, patients with OHCA not witnessed $(N=79,208)$, patients not treated by EMS providers $(N=$ $4,063)$, patients with cardiac arrests occurring during ambulance transport $(N=7,203)$, patients treated by EMS teams without any level-1 EMTs capable of advanced airway placement $(N=5,756)$, traumatic cause $(N=5,062)$ and patients with unknown outcome (prehospital ROSC, survival and neurologic outcome) ( $\mathrm{N}=98)$ and were excluded (Figure 1).

After serial exclusion, 40,443 OHCA patients were included in the final analysis. Regarding the presumed cause of arrest, $36,404(90.0 \%)$ cases were cardiac, 3,050 (7.5\%) were respiratory and 989 (2.4\%) were others. The demographic characteristics according to cause are summarized in Table 1. The proportion of advanced airway placement differed according to the group, and the rate of endotracheal intubation was highest in the respiratory cause group $(6.7 \%$ vs. $8.1 \%$ vs. $5.1 \%$, respectively, $\mathrm{p}<0.01$ ). The neurologically favorable survival-to-discharge rates were $12.5 \%$ in the cardiac group, $9.6 \%$ in the respiratory group, and $12.1 \%$ in the others group (Table $1, \mathrm{p}<0.01$ ).

In the multivariable logistic regression analysis, there was no statistically significant association between type of airway management and good neurologic recovery (SGA as reference, aOR (95\% Cl) 1.01 (0.93-1.11) for BVM; 0.96 (0.81-1.14) for $\mathrm{ETI})$ and survival to discharge (SGA as reference, aOR $(95 \% \mathrm{Cl}) 0.96(0.89-1.03)$ for $\mathrm{BVM}$; $0.96(0.83-1.11)$ for $\mathrm{ETI})$ in the total population.

In the interaction analysis, the magnitude of the association between type of advanced airway and outcome measures was different according to the presumed cause of cardiac arrest. ETI was associated with significantly better neurological recovery than SGA (aOR 3.12 (95\% Cl 1.24-7.80)) and BVM(aOR 3.16 (95\% Cl 1.43-6.98)) when the cause of arrest was respiratory. Although the effect of ETI was nonsignificant, BVM was associated with lower survival to discharge than SGA when the cause of arrest was respiratory (aOR 0.70 (95\% Cl 0.52-0.94)) (Table 3 ).

\section{Discussion}

In this analysis of a nationwide, population-based OHCA registry, we evaluated the association between prehospital advanced airway methods and clinical outcome of cardiac arrest. The magnitude of the association was different according to the presumed cause of cardiac arrest. There was no statistically significant difference in the effect according to the advanced airway type in total population and arrest with cardiac origin. However endotracheal intubation was associated with better neurologic outcome when the cause of arrest was respiratory. We believe more efficient oxygenation and ventilation through prehospital endotracheal intubation provides a rapid and effective solution to respiratory cause of cardiac arrest, as an antidote for drug intoxication. Although most of cardiac arrest cases receive endotracheal intubation eventually after hospital transport, faster reversal of hypoxia and hypoventilation by prehospital endotracheal intubation in the field might show an effect on minimizing hypoxic brain injury. Based on the results of our study, we suggest that 
endotracheal intubation should be considered as a first choice of method in OHCA patients whose cause of arrest is suspected to be respiratory, such as respiratory disease, asphyxia and strangulation.

Even after recent prospective clinical trials, defining the optimal choice of prehospital advanced airway methods in OHCA is controversial. Wang et al. compared laryngeal tube and ETI as prehospital airway management methods for OHCA and showed that LT was superior to ETI when measuring 72-hour survival.(13) Benger et al. compared l-gel versus ETI, and there was no difference in survival to discharge or favorable neurologic outcome rate.(22) Jabre et al. compared BVM to ETI and obtained inconclusive results regarding 28-day survival with favorable neurologic outcome. (12) Overall, the results showed no definite benefit of ETI over BVM or SGA. In a nationwide cohort study conducted in Japan, advanced airway management (ETI and SGA) was associated with better survival and more favorable neurologic outcomes compared with no advanced airway management in non-shockable (pulseless electrical activity and asystole) OHCA patients. (23) However, these studies did not consider the etiologies of arrest.

The supraglottic airway can be inserted blindly and therefore has a higher success rate than endotracheal intubation, even for those with low skills, such as EMS providers in Korea. However, compression of important structures such as carotid vessels has been suggested in animal studies in some SGAs due to its morphologic characteristics and mechanism. (24, 25) In severe hypoxia in respiratory arrest, carotid blood flow reduction caused by SGA insertion might be more critical than for other etiologies of cardiac arrest. One case reported ventilation failure of a drowned patient with supraglottic airways due to leakage from high pressure. (26)

Several previous studies have reported negative effects of prehospital advanced airway placement on the outcome of respiratory cardiac arrest. $(15,18)$ However, these studies included only limited number of patients with medical intrinsic respiratory disease, excluding external causes of arrest, such as asphyxia or hanging. Additionally, the studies only tested the overall effect of prehospital advanced airway rather than the effect of each advanced airway method according to different etiologies, as in our study.

Only witnessed cardiac arrests were enrolled for analysis in our study. We opted not to include patients with arrest not witnessed by initial bystanders. Although our study was a retrospective observational study and there are currently no guidelines for EMS providers to consider cause of arrest when selecting the airway management method, we supposed it might be difficult for EMS providers to estimate the cause of arrest in unwitnessed cases. We believe that, for witnessed cardiac arrest, EMS providers can presume the cause of cardiac arrest based on a history taken from the bystander who witnessed the arrest or could at least determine whether the cause was respiratory or not. Based on the result of our study, we suggest that EMS providers might consider estimated cause when deciding the airway management method in the field.

There are some limitations to our study. First, this was a retrospective observational study; therefore, careful interpretation is needed considering the unique characteristics of our EMS system. Second, we extracted cause of arrest by medical record review. For patients who did not achieve any ROSC and were pronounced dead after initial resuscitation, there might be few clues to estimate the cause of arrest.

Third, the number of patients with favorable neurological outcome in the ETI group with respiratory arrest were very small (9 patients), which leading to wide confidence intervals and making results difficult to interpret. Furthermore, detailed diagnosis of respiratory disease or severity of trauma were not included in our database. Last, there might be unmeasured confounders not included in our registry that could affect both airway management method and outcome.

\section{Conclusion}

The association between prehospital advanced airway and the neurological outcomes of patients with witnessed nontraumatic OHCA was not significant. However, endotracheal intubation was associated with better neurologic recovery in 
cardiac arrest with respiratory cause. Endotracheal intubation may be considered initially by EMS providers when a respiratory cause is suspected for the pathogenesis of cardiac arrest.

\section{Declarations}

\section{CONFLICT OF INTEREST}

The Authors declare that there are no conflicts of interest.

\section{Funding Acknowledgement}

None.

\section{Author Contributions:}

Drs. Choi and Kim had full access to all of the data in the study and take responsibility for the integrity of the data as well as the accuracy of the data analysis.

Study concept and design: Drs. Choi, Kim and Shin

Acquisition, analysis, or interpretation of data: Drs. Kim, Hong and Song

Drafting of the manuscript: Dr. Choi

Critical revision of the manuscript for important intellectual content: Drs. Kim, Song, and Shin

Statistical analysis: Dr. Kim and Choi

Administrative, technical, or material support: Drs. Song and Shin

Study supervision: Drs. Shin, Song and Kim

Manuscript approval: All authors

\section{References}

1. Moriwaki Y, Tahara Y, Kosuge T, Suzuki N. Etiology of out-of-hospital cardiac arrest diagnosed via detailed examinations including perimortem computed tomography. J Emerg Trauma Shock. 2013;6(2):87-94.

2. Engdahl J, Holmberg M, Karlson BW, Luepker R, Herlitz J. The epidemiology of out-of-hospital 'sudden' cardiac arrest. Resuscitation. 2002;52(3):235-45.

3. Safar P, Paradis N, Weil M. Asphyxial cardiac arrest. Cardiac arrest The science and practice of resuscitation medicine. 1996;39:702-26.

4. Truhlar A, Deakin CD, Soar J, Khalifa GE, Alfonzo A, Bierens JJ, et al. European Resuscitation Council Guidelines for Resuscitation 2015: Section 4. Cardiac arrest in special circumstances. Resuscitation. 2015;95:148-201.

5. Richman PB, Nashed AH. The etiology of cardiac arrest in children and young adults: special considerations for ED management. Am J Emerg Med. 1999;17(3):264-70.

6. Brierley JB. Experimental hypoxic brain damage. J Clin Pathol Suppl (R Coll Pathol). 1977;11:181-7.

7. Erecinska M, Silver IA. Tissue oxygen tension and brain sensitivity to hypoxia. Respiration Physiology. 2001;128(3):26376.

8. Ong MEH, Perkins GD, Cariou A. Out-of-hospital cardiac arrest: prehospital management. The Lancet: Elsevier; 2018. p. 980-8. 
9. Alexander R, Hodgson P, Lomax D, Bullen C. A comparison of the laryngeal mask airway and Guedel airway, bag and facemask for manual ventilation following formal training. Anaesthesia. 1993;48(3):231-4.

10. Kurola J, Harve H, Kettunen T, Laakso JP, Gorski J, Paakkonen H, et al. Airway management in cardiac arrest-comparison of the laryngeal tube, tracheal intubation and bag-valve mask ventilation in emergency medical training. Resuscitation. 2004;61(2):149-53.

11. Andersen LW, Granfeldt A. Pragmatic Airway Management in Out-of-Hospital Cardiac Arrest. Jama2018. p. 761-3.

12. Jabre P, Penaloza A, Pinero D, Duchateau F-X, Borron SW, Javaudin F, et al. Effect of Bag-Mask Ventilation vs Endotracheal Intubation During Cardiopulmonary Resuscitation on Neurological Outcome After Out-of-Hospital Cardiorespiratory Arrest: A Randomized Clinical Trial. Jama2018. p. 779-87.

13. Wang HE, Schmicker RH, Daya MR, Stephens SW, Idris AH, Carlson JN, et al. Effect of a Strategy of Initial Laryngeal Tube Insertion vs Endotracheal Intubation on 72-Hour Survival in Adults With Out-of-Hospital Cardiac Arrest: A Randomized Clinical Trial. Jama2018. p. 769-78.

14. Wang HE, Benger JR. Endotracheal intubation during out-of-hospital cardiac arrest: New insights from recent clinical trials. Journal of the American College of Emergency Physicians Open: John Wiley \& Sons, Ltd; 2019.

15. Ohashi-Fukuda N, Fukuda T, Yahagi N. Effect of Pre-Hospital Advanced Airway Management for Out-Of-Hospital Cardiac Arrest Caused by Respiratory Disease: A Propensity Score-Matched Study. Anaesthesia and Intensive Care2017. p. 375-83.

16. Orban JC, Truc M, Kerever S, Novain M, Cattet F, Plattier R, et al. Comparison of presumed cardiac and respiratory causes of out-of-hospital cardiac arrest. Resuscitation. 2018;129:24-8.

17. Panchal AR, Bobrow BJ, Spaite DW, Berg RA, Stolz U, Vadeboncoeur TF, et al. Chest compression-only cardiopulmonary resuscitation performed by lay rescuers for adult out-of-hospital cardiac arrest due to non-cardiac aetiologies. Resuscitation. 2013;84(4):435-9.

18. Fukuda T, Fukuda-Ohashi N, Doi K, Matsubara T, Yahagi N. Effective pre-hospital care for out-of-hospital cardiac arrest caused by respiratory disease. Heart, lung \& circulation2015. p. 241-9.

19. Ro YS, Shin SD, Song KJ, Lee EJ, Kim JY, Ahn KO, et al. A trend in epidemiology and outcomes of out-of-hospital cardiac arrest by urbanization level: A nationwide observational study from 2006 to 2010 in South Korea. Resuscitation: Elsevier; 2013. p. 547-57.

20. Kim YT, Shin SD, Hong SO, Ahn KO, Ro YS, Song KJ, et al. Effect of national implementation of utstein recommendation from the global resuscitation alliance on ten steps to improve outcomes from Out-of-Hospital cardiac arrest: a ten-year observational study in Korea. BMJ Open: British Medical Journal Publishing Group; 2017.

21. Perkins GD, Jacobs IG, Nadkarni VM, Berg RA, Bhanji F, Biarent D, et al. Cardiac arrest and cardiopulmonary resuscitation outcome reports: update of the Utstein Resuscitation Registry Templates for Out-of-Hospital Cardiac Arrest: a statement for healthcare professionals from a task force of the International Liaison Committee on Resuscitation (American Heart Association, European Resuscitation Council, Australian and New Zealand Council on Resuscitation, Heart and Stroke Foundation of Canada, InterAmerican Heart Foundation, Resuscitation Council of Southern Africa, Resuscitation Council of Asia); and the American Heart Association Emergency Cardiovascular Care Committee and the Council on Cardiopulmonary, Critical Care, Perioperative and Resuscitation. Circulation. 2015;132(13):1286-300.

22. Benger JR, Kirby K, Black S, Brett SJ, Clout M, Lazaroo MJ, et al. Effect of a Strategy of a Supraglottic Airway Device vs Tracheal Intubation During Out-of-Hospital Cardiac Arrest on Functional Outcome: The AIRWAYS-2 Randomized Clinical Trial. Jama2018. p. 779-91.

23. Izawa J, Komukai S, Gibo K, Okubo M, Kiyohara K, Nishiyama C, et al. Pre-hospital advanced airway management for adults with out-of-hospital cardiac arrest: Nationwide cohort study. BMJ (Online): BMJ Publishing Group; 2019. 
24. Kim TH, Hong KJ, Shin SD, Lee JC, Choi DS, Chang I, et al. Effect of endotracheal intubation and supraglottic airway device placement during cardiopulmonary resuscitation on carotid blood flow over resuscitation time: An experimental porcine cardiac arrest study. Resuscitation. 2019;139:269-74.

25. Segal N, Yannopoulos D, Mahoney BD, Frascone RJ, Matsuura T, Cowles CG, et al. Impairment of carotid artery blood flow by supraglottic airway use in a swine model of cardiac arrest. Resuscitation. 2012;83(8):1025-30.

26. Baker PA, Webber JB. Failure to ventilate with supraglottic airways after drowning. Anaesth Intensive Care. 2011;39(4):675-7.

\section{Tables}


Table 1

Demographic and clinical characteristics according to etiology of arrest

\begin{tabular}{|c|c|c|c|c|c|c|c|c|c|}
\hline & \multicolumn{2}{|l|}{ Total } & \multicolumn{2}{|l|}{ Cardiac } & \multicolumn{2}{|c|}{ Respiratory } & \multicolumn{3}{|l|}{ Miscellaneous } \\
\hline & $\mathrm{N}$ & $\%$ & $\mathrm{~N}$ & $\%$ & $\mathrm{~N}$ & $\%$ & $\mathrm{~N}$ & $\%$ & $\begin{array}{l}\mathrm{p}- \\
\text { value }\end{array}$ \\
\hline Total & 40,443 & & 36,404 & 90.0 & 3,050 & 7.5 & 989 & 2.4 & \\
\hline Age (mean (SD), years) & \multicolumn{2}{|c|}{$68.48(15.96)$} & \multicolumn{2}{|c|}{$68.86(15.59)$} & \multicolumn{2}{|c|}{$66.95(18.57)$} & \multicolumn{2}{|l|}{$59.23(17.39)$} & $\begin{array}{l}<.001 \\
0.001\end{array}$ \\
\hline Male & 25,925 & 64.1 & 23,334 & 64.1 & 1,886 & 61.8 & 705 & 71.3 & $\begin{array}{l}< \\
0.001\end{array}$ \\
\hline \multicolumn{10}{|l|}{ Past medical history } \\
\hline $\begin{array}{l}\text { Heart } \\
\text { disease }\end{array}$ & 6,555 & 16.2 & 6,297 & 17.3 & 192 & 6.3 & 66 & 6.7 & $\begin{array}{l}<.001 \\
0.001\end{array}$ \\
\hline Hypertension & 13,290 & 32.9 & 12,361 & 34.0 & 754 & 24.7 & 175 & 17.7 & $\begin{array}{l}< \\
0.001\end{array}$ \\
\hline $\begin{array}{l}\text { Diabetes } \\
\text { mellitus }\end{array}$ & 8,427 & 32.9 & 7,811 & 21.5 & 509 & 16.7 & 107 & 10.8 & $\begin{array}{l}< \\
0.001\end{array}$ \\
\hline Stroke & 3,673 & 9.1 & 3,290 & 9.0 & 347 & 11.4 & 36 & 3.6 & $\begin{array}{l}< \\
0.001\end{array}$ \\
\hline \multicolumn{10}{|l|}{ Circumstance of arrest, } \\
\hline Private place & 31,090 & 76.9 & 28,223 & 77.5 & 2,296 & 75.3 & 571 & 57.7 & $\begin{array}{l}< \\
0.001\end{array}$ \\
\hline $\begin{array}{l}\text { Bystander } \\
\text { CPR }\end{array}$ & 25,331 & 62.6 & 22,818 & 62.7 & 1,961 & 64.3 & 552 & 55.8 & $\begin{array}{l}<.001 \\
0.001\end{array}$ \\
\hline $\begin{array}{l}\text { Airway } \\
(\%)\end{array}$ & & & & & & & & & $\begin{array}{l}< \\
0.001\end{array}$ \\
\hline SGA & 11,460 & 28.3 & 10,500 & 28.8 & 736 & 24.1 & 224 & 22.6 & \\
\hline BVM & 26,244 & 64.9 & 23,462 & 64.4 & 2,067 & 67.8 & 715 & 72.3 & \\
\hline ETI & 2,739 & 6.8 & 2,442 & 6.7 & 247 & 8.1 & 50 & 5.1 & \\
\hline \multicolumn{10}{|l|}{$\begin{array}{l}\text { Elapsed time (median } \\
\text { (IQR), min) }\end{array}$} \\
\hline $\begin{array}{l}\text { Response } \\
\text { time interval }\end{array}$ & \multicolumn{2}{|c|}{$7.0(5.0-10.0)$} & \multicolumn{2}{|c|}{$7.0(5.0-9.0)$} & \multicolumn{2}{|c|}{$7.0(5.0-10.0)$} & \multicolumn{2}{|l|}{$7.0(5.0-11.0)$} & $<.001$ \\
\hline $\begin{array}{l}\text { Scene time } \\
\text { interval }\end{array}$ & \multicolumn{2}{|c|}{$\begin{array}{l}10.0(8.0- \\
15.0)\end{array}$} & \multicolumn{2}{|c|}{$\begin{array}{l}11.0(8.0- \\
15.0)\end{array}$} & \multicolumn{2}{|c|}{$10.5(7.0-15.0)$} & \multicolumn{2}{|l|}{$8.0(6.0-14.0)$} & $\begin{array}{l}<.001 \\
0.001\end{array}$ \\
\hline \multicolumn{10}{|l|}{ Post resuscitation care } \\
\hline Reperfusion & 2,511 & 6.2 & 2,476 & 6.8 & 15 & 0.5 & 20 & 2.0 & $\begin{array}{l}< \\
0.001\end{array}$ \\
\hline Hypothermia & 1,762 & 4.4 & 1,505 & 4.1 & 195 & 6.4 & 62 & 6.3 & $\begin{array}{l}< \\
0.001\end{array}$ \\
\hline
\end{tabular}

CPR: cardiopulmonary resuscitation; SGA: supraglottic airway; ETI: endotracheal intubation; BVM: bag valve mask; ECMO: extracorporeal membrane oxygenation; Good CPC: CPC 1 or 2. 


\begin{tabular}{|c|c|c|c|c|c|c|c|c|c|}
\hline \multirow[b]{2}{*}{ ECMO } & \multicolumn{2}{|l|}{ Total } & \multicolumn{2}{|c|}{ Cardiac } & \multicolumn{2}{|c|}{ Respiratory } & \multicolumn{3}{|c|}{ Miscellaneous } \\
\hline & 505 & 1.2 & 483 & 1.3 & 13 & 0.4 & 9 & 0.9 & $\begin{array}{l}<.001 \\
0.01\end{array}$ \\
\hline \multicolumn{10}{|l|}{ Outcome } \\
\hline $\begin{array}{l}\text { Survival to } \\
\text { discharge }\end{array}$ & 4,965 & 12.3 & 4,551 & 12.5 & 294 & 9.6 & 120 & 12.1 & $\begin{array}{l}<.001 \\
0 .\end{array}$ \\
\hline Good CPC & 3,188 & 7.9 & 3,057 & 8.4 & 60 & 2.0 & 71 & 7.2 & $\begin{array}{l}<.001 \\
0.00\end{array}$ \\
\hline
\end{tabular}

\section{Table 2}

Multivariable logistic regression analysis by type of prehospital airway management method

\begin{tabular}{|c|c|c|c|c|c|c|c|c|c|c|c|}
\hline & \multirow{3}{*}{$\begin{array}{l}\text { Total } \\
\mathrm{N}\end{array}$} & \multicolumn{5}{|c|}{ Good neurological outcome } & \multicolumn{5}{|l|}{$\begin{array}{l}\text { Survival to } \\
\text { discharge }\end{array}$} \\
\hline & & \multirow[b]{2}{*}{$\mathrm{n}(\%)$} & \multicolumn{2}{|c|}{ Unadjusted } & \multicolumn{2}{|c|}{ Adjusted } & \multirow[b]{2}{*}{$\mathrm{n}(\%)$} & \multicolumn{2}{|c|}{ Unadjusted } & \multicolumn{2}{|c|}{ Adjusted } \\
\hline & & & OR & $95 \% \mathrm{Cl}$ & OR & $95 \% \mathrm{Cl}$ & & OR & $95 \% \mathrm{Cl}$ & OR & $95 \% \mathrm{Cl}$ \\
\hline All & 40,443 & $\begin{array}{l}3,188 \\
(7.9)\end{array}$ & & & & & $4,965(12.3)$ & & & & \\
\hline SGA & 11,460 & $\begin{array}{l}974 \\
(8.5)\end{array}$ & 1.00 & & & & $\begin{array}{l}1,540 \\
(13.4)\end{array}$ & 1.00 & & & \\
\hline BVM & 26,244 & $\begin{array}{l}2,015 \\
(7.7)\end{array}$ & 0.89 & $\begin{array}{l}(0.73- \\
0.97)\end{array}$ & 1.01 & $\begin{array}{l}(0.93- \\
1.11)\end{array}$ & $\begin{array}{l}3,090 \\
(11.8)\end{array}$ & 0.86 & $\begin{array}{l}(0.81- \\
0.92)\end{array}$ & 0.96 & $\begin{array}{l}(0.89- \\
1.03)\end{array}$ \\
\hline ETI & 2,739 & $\begin{array}{l}199 \\
(7.3)\end{array}$ & 0.84 & $\begin{array}{l}(0.72- \\
0.99)\end{array}$ & 0.96 & $\begin{array}{l}(0.81- \\
1.14)\end{array}$ & $\begin{array}{l}335 \\
(12.2)\end{array}$ & 0.90 & $\begin{array}{l}(0.79- \\
1.02)\end{array}$ & 0.96 & $\begin{array}{l}(0.83- \\
1.11)\end{array}$ \\
\hline
\end{tabular}


Table 3

Multivariable logistic regression with interaction analysis between airway management methods and the etiology

\begin{tabular}{|c|c|c|c|c|c|c|}
\hline & \multicolumn{3}{|c|}{ Good neurological outcome } & \multicolumn{3}{|c|}{ Survival to discharge } \\
\hline & $\mathrm{N}(\%)$ & $\mathrm{aOR}$ & $95 \% \mathrm{Cl}$ & $\mathrm{N}(\%)$ & $\mathrm{aOR}$ & $95 \% \mathrm{Cl}$ \\
\hline \multicolumn{7}{|l|}{ Cardiac } \\
\hline $\operatorname{SGA}(n=10,500)$ & $942(9.0)$ & 1.00 & & $1,425(13.6)$ & 1.00 & \\
\hline $\operatorname{BVM}(n=23,462)$ & $1,929(8.2)$ & 1.02 & $(0.93-1.11)$ & $2,827(12.0)$ & 0.96 & $(0.89-1.03)$ \\
\hline $\mathrm{ETI}(\mathrm{n}=2,442)$ & $186(7.6)$ & 0.92 & $(0.77-1.10)$ & $299(12.2)$ & 0.96 & $(0.83-1.11)$ \\
\hline \multicolumn{7}{|l|}{ Respiratory } \\
\hline SGA $(n=736)$ & $13(1.8)$ & 1.00 & & $84(11.4)$ & 1.00 & \\
\hline $\operatorname{BVM}(n=2,067)$ & $38(1.8)$ & 0.99 & $(0.51-1.91)$ & $181(8.8)$ & 0.70 & $(0.52-0.94)$ \\
\hline ETI $(n=247)$ & $9(3.6)$ & 3.12 & $(1.24-7.80)$ & $29(11.7)$ & 1.35 & $(0.83-2.18)$ \\
\hline \multicolumn{7}{|l|}{ Miscellaneous } \\
\hline $\operatorname{SGA}(n=224)$ & $19(8.5)$ & 1.00 & & $31(13.8)$ & 1.00 & \\
\hline BVM $(n=715)$ & $48(6.7)$ & 0.98 & $(0.31-3.11)$ & $82(11.5)$ & 1.53 & $(0.61-3.85)$ \\
\hline ETI $(n=50)$ & $4(8.0)$ & 1.15 & $(0.11-11.91)$ & $7(14.0)$ & 1.59 & $(0.28-9.07)$ \\
\hline
\end{tabular}

\section{Figures}




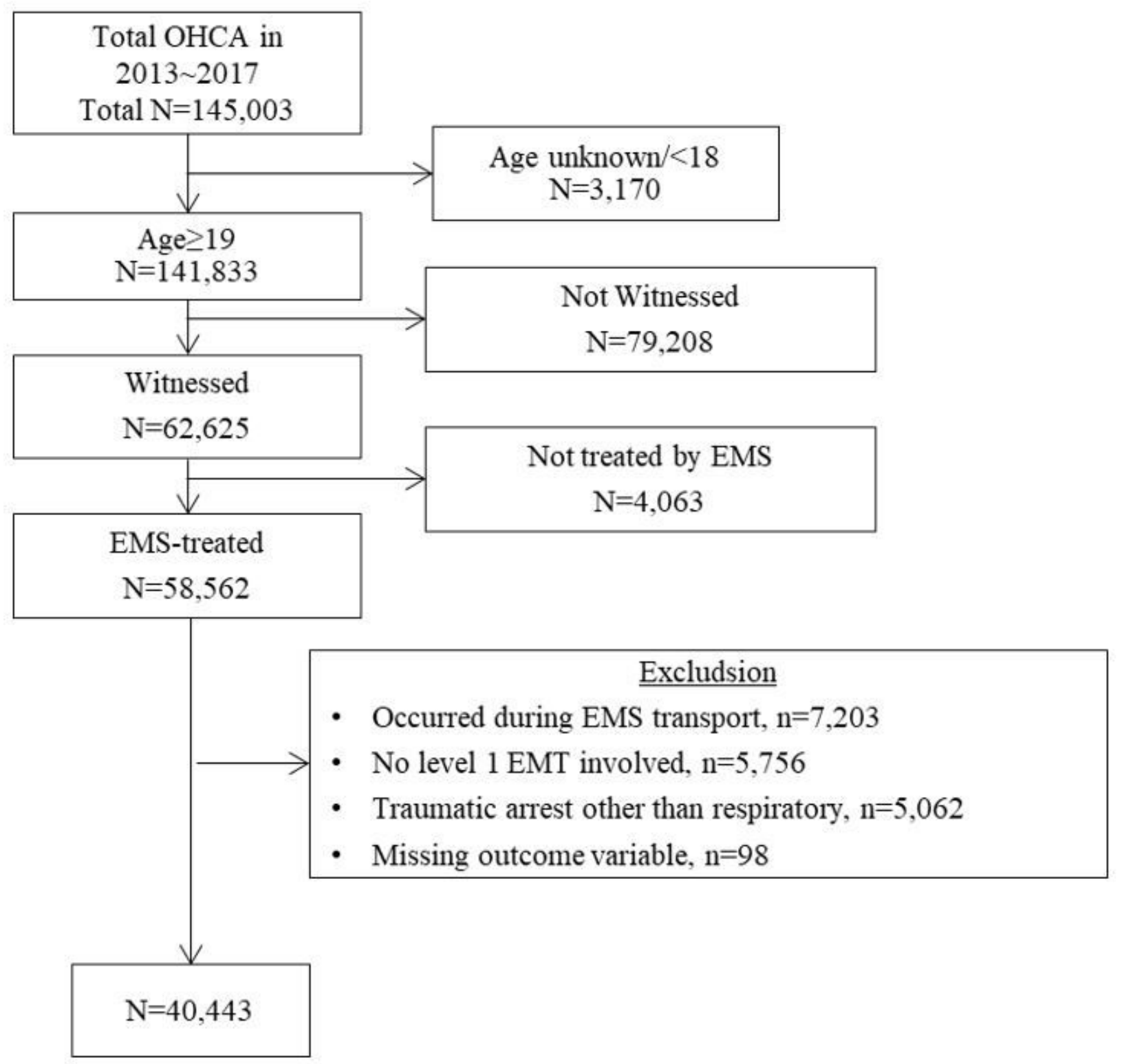

Figure 1

Enrollment flow of study population ; OHCA: out-of-hospital cardiac arrest; EMS: emergency medical service ; EMT; emergency medical technician 